\title{
Determination and Clinical Significance of Various Plasma Lipoprotein Pattern
}

\author{
Seidel* \\ Department of Clinical Chemistry, Medizinische \\ Universitätsklinik Heidelberg, Germany
}

Prospective epidemiologic studies in different countries have uniformly demonstrated that a high plasma cholesterol level is predictive of an increased risk for myocardial infarction. However, one has to be careful to avoid the simplistic view that serum cholesterol level alone is the only determinant of atherogenesis. Beside elevated serum lipids diabetes, smoking, hypertension, obesity and stress of live have also been correlated with a higher incidence of atherosclerosis. As atherosclerosis is now the greatest single cause of premature death in nearly all highly industrialized countries, it is evident that the clinician who is actively involved in clinical investigation finds lipid and lipoprotein metabolism a subject of particular interest. Among all the different molecular diseases of lipid and lipoprotein metabolism certainly hyperlipidemias are the most common disorders the clinician has to deal with.

The importance of plasma lipids and the rapid development of new, simple and efficient analytical tools for their separation and determination have provided important stimuli for many qualitative and quantitative studies of the various lipid components of plasma lipoprotein classes.

While these studies have contributed significantly to our knowledge of the lipid composition of lipoproteins in health and disease, only recently adaquate information regarding the composition and nature of protein moieties ("apolipoproteins") became available. The re-

* Asist Professor of Internal Medicine Chief of Division of Lipid Research University of Heidlberg, West Germany cognition of the importance of protein moieties, however, for the structural stability and the specifity of plasma lipoproteins and their distribution within the individual lipoprotein classes, have emphasized the need for better understanding of these aspects of lipid metabolism and lipoprotein chemistry.

The classification and nomenclature in the lipoprotein field has been commonly based on two sets of operational terms. Differences in the hydrated density of plasma lipoproteins which are largely a consequence of their lipid content and differences in their electrophoretic mobility, which is primarily a consequence of their protein moieties, have provided the basis of a widely accepted classification system comprising four major classes, each of which is characterized by a relatively narrow density range and a distinguished electrophoretic band.

1) The chylomicrons within the density range d $0.9-0.95 \mathrm{~g} / \mathrm{ml}$ consisting of $1 \%$ protein, $4 \%$ phospholipids, 6\% cholesterol and $85-90 \%$ triglycerides of exogenous origin.

2) The very low density lipoproteins within the density range

d $0.95-1.006 \mathrm{~g} / \mathrm{ml}$ consisting of $8-10 \%$ protein, $18 \%$ phospholipids, $19 \%$ cholesterol and $50 \%$ triglycerides of endogenous origin.

3) The low density lipoproteins within the density range

d $1.006-1.063 \mathrm{~g} / \mathrm{ml}$ consisting of $20 \%$ protein, $23 \%$ phospholipids, $45 \%$ cholesterol and $10 \%$ triglycerides.

4) The high density lipoproteins within the density range

d $1.063-1.121 \mathrm{~g} / \mathrm{ml}$ consisting of $50 \%$ pro- 
tein, 30\% phospholipids, $18 \%$ cholesterol and $2-5 \%$ triglycerides.

Qualitative and quantitative differences in the protein moieties of the lipoproteins lead to four different bands on electrophoresis.

1) The non migrating chylomicrons.

2) The $\beta$-lipoproteins migrating like the $\beta$-globulins representing the LDL fraction.

3) The pre- $\beta$ lipoproteins migrating like the $\alpha_{2}$-globulins corresponding with the VLDL fraction.

4) The $\alpha$-lipoproteins corresponding with the HDL fraction and migrating with the mobility of the $\alpha_{1}$-globulins.

These classes of lipoproteins are, however, polydisperse systems, heterogenous with respect to particle size, hydrated density and apolipoproteins.
Three distinct protein moieties have been identified in the spectrum of human serum lipoproteins differently distributed over the spectrum and designated apolipoprotein $\mathrm{A}$, apolipoprotein B and apolipoprotein C. They differ in their immunological properties, molecular weight, $\mathrm{N}$-and $\mathrm{C}$-terminal amino acids, amino acid composition, sedimentation coefficient and carbohydrate content. There is increasing evidence for move, not yet fully identified apolipoproteins.

\section{Apo A}

The apolipoprotein $\mathrm{A}$ is the major protein moiety of the HDL fraction, but is also found in the protein moiety of the VLDL fraction where it is believed to cause the $\alpha_{2}$ mobility of

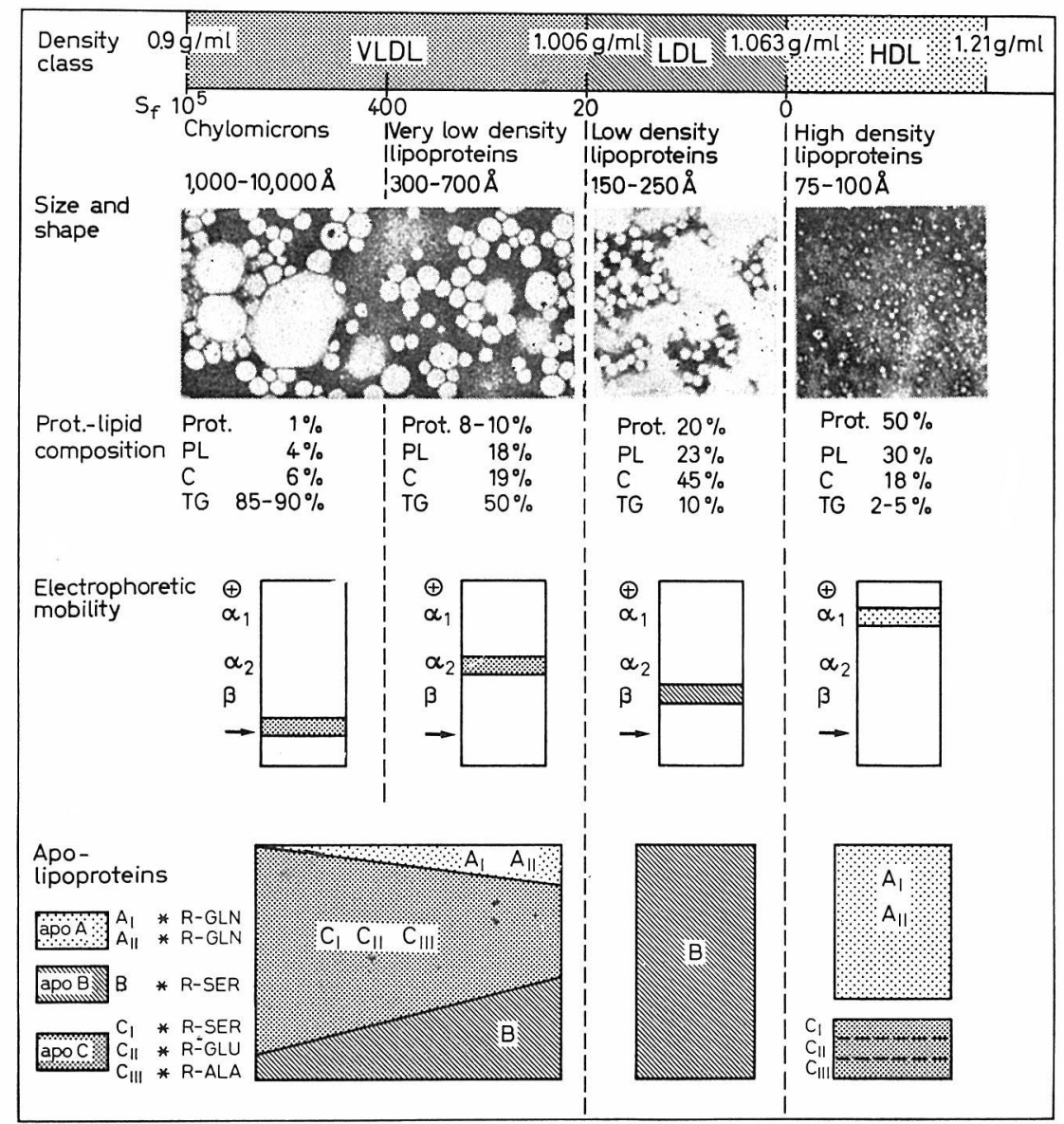

Fig.1 Characteristics of human plasma lipoproteins. 
the pre- $\beta$ lipoproteins.

Very recently it has been reported by several groups of investigators that apolipoprotein $\mathrm{A}$ consists of two subunits designated A-1 and A-II differing in their primary structure and immunologic properties. Both peptides seem to show glutamin as C-terminal amino acid. Further studies on the characterization of the HDL fraction have extended these observations and it was demonstrated that different subfractions of HDL showed not only significant differences in their lipid composition but also differences in their A-1/A-II ratio. Although both of the apo A peptides are capable to bind lipids it seems as if both of them are essentially for the quarternary structure of normal $\alpha$ lipoproteins. Recently it was demonstrated that the A-I peptide acts as a cofactor for the lecithin: cholesterol acyltransferase, the LCATenzyme.

\section{Apo B}

Apo B represents the major protein moiety of the LDL fraction from normals. But it also accounts for $40 \%$ of the protein moiety of the VLDL fraction. After total delipidization it is insoluble in water or in urea and only recently methods have been described to solubilize the apolipoprotein. Therefore little is known about the physical-chemical properties of apolipoprotein B. Serin appears to be the only Cterminal, glutamic acid the only N-terminal amino acid. The amino acid composition of apo $B$ is significantly different from that of the two apo A peptides. The carbohydrate content of apo B is somewhat higher than that of the apo A.

Immunochemically apo B seems to consist of only one peptide, however, several inherited antigenic polymorphic systems have been reported.

\section{Apo C}

The protein moiety of the VLDL fraction consists of apo A, apo B and Apo C. The soluble protein moiety of a totally delipidized VLDL fraction accounts for $60 \%$ and can be separated by gel filtration into two major fractions. One fraction consists of apo A polypeptides accounting for less than $10 \%$, whereas the second protein fraction, designated apolipoprotein $\mathrm{C}$ accounts for $50 \%$ of the total VLDL protein. Apo $\mathrm{C}$ is a mixture of three polypeptides characterized by serine and threonine as N-terminal amino acids, which can be separated by a DEAE cellulose column chromatography. On the basis of their Cterminal amino acids these apo $\mathrm{C}$ peptides have been designated C-I (R-Ser), C-II (R-Glu) and C-III (R-Ala).

Although the apo $\mathrm{C}$ is the major protein moiety of the chylomicrons and of the pre- $\beta$ lipoprotein fraction, traces of apo $\mathrm{C}$ have also been found in the HDL fraction, namely the C-II (R-Glu), designated peptide, which acts as a cofactor for the lipoproteinlipase. The C-III peptide in contrast seems to inhibit at least part of the postheparin lipolytic activity.

The increasing importance of the apolipoproteins for maintaining the structure of lipoproteins and, therefore, their role in lipid metabolism become more and more apparent. Although the primary structure of some of the peptides has been determined we still don't know what causes the apolipoproteins to bind their lipids. A very important question to be ansered! The different apolipoproteins possess a different capacity to bind small amounts of fatty acids and phospholipids covalently. Such chemical compositions may be of a highly lipophilic character and, therefore, and important determinant for the final protein lipid composition of the individual lipoprotein. In addition, the protein-carbohydrate bounds on the apolipoproteins and the carbohydrate moieties themselves may play a similar role. The slightest modifications of the structure of the apolipoproteins as well as a change in the ratios between the apolipoproteins forming the protein portion of normal lipoprotein families may lead to lipoproteins of altered physicalchemical properties.

Since we know more about the protein moieties of the plasmalipoproteins and their distribution between the different classes, no one of the two classification systems based on density, or electro-phoretic mobility, is any- 
more adequate to describe the different classes of lipoproteins from a physiological-chemical point of view.

Therefore, a new classification system has been proposed based on the protein portion as the essential compound of lipoprotein particles with regard to their structure and lipid composition. It should, however, be pointed out that the application of such a concept will, in the density range above $\mathrm{d} 1.01 \mathrm{~g} / \mathrm{ml}$, result in products containing more than one apolipoprotein. The concepseems to be justified, however, chemically and biologically. Chemically, it utilizes unique chemical components, the apolipoproteins, as criteria for differentiation of various lipoprotein families. It recognizes the capacity and limitation of each apolipoprotein to bind various amounts of lipids. Biologically, it may point out differences in the metabolic origin and fate of various lipoprotein families, the later becomes even more apparent since we know about the different actions of some of the apolipoprotein peptides upon the lipolytic enzymes. Geretic disorders of lipid transport such as Tangier disease and abetalipoproteinaemia are characterized primarily by the absence of an apolipoprotein and only secondarily by a decrease of a specific density class or an electrophoretic band. The recent observation that a decrease of dog plasma cholesterol levels by androgen administration could be correlated with the specific effect of this hormone on the rate of synthesis of apolipoprotein $\mathrm{A}$ is another excellent example of a successful application of this concept to study lipid metabolism and the action of lipid-lowering agents.

The importance of such a classification system has been proved useful for the understanding of abnormal plasma lipid concentrations. The careful analysis of the lipoproteins revealed abnormalities of their protein moieties in many cases, particularin secondary forms of dyslipoproteinemia, how of which I should like to discuss later in this presentation.

Both primary and secondary hyperlipidemias represent such a variety of abnormalities that an internationally acceptable 'classification was necessary for communication. Based on lipoprotein analysis the so called "typing sys- tem" has offered such a possibility.-

Today the primary forms of hyperlipoproteinemia, as you all know, are divided into 5 types. I should now like to describe briefly some of the features of these familial types. Although we have to recognice that this typing system will eventually be further modifical as our knowledge about dyslipoproteinemia increases.

\section{Type I hyperlipoproteinaemia}

Type I hyperlipoproteinaemia is characterised by the presence of chylomicrons in a patient's fasting plasma. Fasting plasma normally does not contain chylomicrons. This disorder is mainly seem by the paediatrician. Typically a baby or a child develops attacks of abdominal pain. Crops of eruptive xanthomata may oceur and lipaemia retinalis may be observed. The plasma is markedly lipaemic due to chylomicrons, the presence of which is reflected by the often enormous increase of triglycerides which typically rise to 3,000 to $10,000 \mathrm{mg}$. per $100 \mathrm{ml}$. Cholesterol levels are proportionately raised; the net effect is a total plasma triglyceride: cholesterol ratio of about 10: 1. Lipoprotein electrophoresis confirms the presence of chylomicrons, as does storing of the plasma overnight at $4^{\circ} \mathrm{C}$. when the chylomicrons will float leaving a clear plasma below.

They chylomicronaemia is thought to be due to the deficiency of the enzyme lipoprotein lipase which initiates the breakdown of these particles. The normal activation, of lipoprotein lipase by intravenous heparin injection, as assessed by an in vitro assay of plasma lipolytic activity, is not found in these subjects.

Correction of the chylomicronaemia is promptly effected by the withdrawal of dietary fat. Within a few days the lipid levels fall and may become almost normal.

\section{Type II hyperlipoproteinaemia}

The lipoprotein phenotype of Type II hyperlipoproteinaemia is characterised by an increase of beta lipoproteins of normal density.

The increase of beta lipoproteins is characterised by an increase in the plasma cholesterol 
level with a normal triglyceride level, the plasma remaining clear. In these cases it is not necessary to have recourse to lipoprotein electrophoresis and ultracentrifugation to establish the diagnosis. In some subjects, however, triglyceride levels are also elevated due to raised pre-beta lipoprotein levels. Therefore recently the type II hyperlipopreteinaemia has been divided into two types: Type II a and Type II b. Both are characterised by strongly elevated beta-lipoproteins and cholesterrol concentrations. While in Type II a the triglycerides and pre-beta-lipoproteins are normal, in Type II b these fractions are also elevated.

The clinical consequences of Type II hyperlipoproteinaemia may vary. Nevertheless, there is no doubt that in many subjects it is associated with the early development of vascular disease.

Recently we were able to demonstrate that the $\beta$-lipoproteins from type II patients show a disk like shape with a strong tendency the aggregate.

Since it is known that the bialogical half live of $\beta$-lipoproteins is as-normally long in type II patients these stractural konges might be due to an ageing effect. it seems possible that this abnormality may be of importance with regard to the filtration and though of the $\beta$-lipoproteins in the arterial wall. Substantial reductions on serum total cholesterol and beta lipoprotein cholesterol levels can be achieved by a low cholesterol diet. The main features consist of elimination of egg yolk in any form, organ meats such als liver and kidney, dairy products such as whole milk, butter and most cheeses, fatty meats and shellfood. Weight reduction diets have no place, per se, in the management of hyperbeta-lipoproteinaemia. Cholestyramine and Nicotinic acid have produced encouraging results in clinical trials by further reducing the cholesterol levels.

However, the ultimate benefit of such treatment remains to be demonstrated.

\section{Type III hyperlipoproteinaemia}

Type III hyperlipoproteinaemia is characterised by the presence of beta lipoproteins of abnormally low density so that it is found together with pre-beta lipoprotein at density $<1.006$. It is generally associated with raised plasma triglyceride and cholesterol levels, and the serum may be lipaemic. The elevation of plasma cholesterol relative to triglyceride levels is greater than that seen in Type IV hyperlipoproteinaemia since the abnormal fraction contains triglyceride and cholesterol in a ratio of about $3: 1$ in contrast to pre-beta lipoprotein with the corresponding ratio of $5: 1$. However, plasma lipid levels may prove an unreliable guide to the presence of Type III hyperlipoproteinaemia as, for example, when high triglyceride levels due to elevated pre-beta lipoproteins are accompanied by a raised beta lipoprotein cholesterol level as is seen in some subjects with Type II hyperlipoproteinaemia. Lipoprotein electrophoresis of the plasma will often show the so-called 'broad beta' band and some chylomicrons may also be seen. The diagnosis is established by obtaining the ultracentrifugal fractions $d<1.006$ and showing it to contain lipoproteins of beta mobility on paper electrophoresis, so-called 'floating' beta.

The protein portion of the abnormal $\beta$ lipoprotein in this disease consists in conternt to normal $\beta$-lipoproteins not only of apo $\beta$ but also of apo $\mathrm{L}$, which in ade an immunological test system possible for the diagnosis at the type III hyperlipoproteinemia without the use of the ultracentrifuge.

The full clinical picture of Type III remains to be defined. Xanthomata and corneal arcus may occur as in Type II hyperlipoproteinaemia. The xanthomata may show an inflamed appearance, when they are called tuboeruptive. Planar xanthomata in the palmar creases may be prominent and almost diagnostic; they also occur in biliary cirrhosis. Type III hyperlipoproteinaemia is undoubtedly much less common than Types II and IV. Nevertheless it is probably not rare.

Type III hyperlipoproteinaemia may be associated with extensive vascular disease.

Treatment of Type III hyperlipoproteinaemia is extremely gratifying. It has 3 components: (1) low cholesterol intake; (2) weight reduction followed by maintenance of a diet in which calories are derived from protein, fat and carbohydrate in a ratio of $20: 40: 40$; (3) 
動脈硬化 Vol. 1

clofibrate 1.5 to $2 \mathrm{~g}$. per day. Lipid levels drop substantially and sometimes to rather low levels and xanthomata regress. Again the long term consequences of treatment remain to be evaluated.

\section{Type IV hyperlipoproteinaemia}

The lipoprotein phenotype is an increase of pre-beta lipoprotein causing raised plasma triglyceride levels without chylomicronaemia. It often occurs as a secondary feature of diseases such as diabetes, hypothyroidism, nephrosis, pancreatitis, etc. It is also frequently seen, howevern, as a primary abnormality, apparently genetically determind.

The lipid pattern is usually that of raised triglyceride and normal or moderately elevated cholesterol levels, reflecting the composition of pre-beta lipoprotein. Depending on the degree of pre-beta lipoprotein increase the plasma may become turbid or lipaemic. This lipaemia is not modified by storage overnight at $4^{\circ} \mathrm{C}$. in contrast to that due to chylomicrons and lipoprotein electrophoresis shows the increased prebeta lipoprotein band to be responsible for the triglyceride increase and not chylomicrons or the abnormal beta lipoprotein of Type III hyperlipoproteinaemia.

Subjects with Type IV hyperlipoproteinaemia are often overweight, and show hyperuricaemia and impaired glucose tolerance. Xanthomata are uncommon but when the hyperlipoproteinaemia is sufficiently severe eruptive lesions occur, as does lipaemia retinalis and attacks of abdominal pain. It is likely that Type IV hyperlipoproteinaemia predisposes to vascular disease of the peripheral type.

The disorder responds well to treatment. Subjects are brought to ideal weifht and this is maintained by the provision of the 20:40:40: diet described for Type III hyperlipoproteinaemia. Clofibrate is effective in most cases.

\section{Type V hyperlipoproteinaemia}

This uncommon disorder features raised plasma triglyceride levels due both to chylo- microns and pre-beta lipoproteins. The trigylceride levels are often quite high, with an elevation of cholesterol which for a given triglyceride level lies between that seen for Types I and IV hyperlipoproteinaemia. The plasma is milky and if stored at $4^{\circ} \mathrm{C}$. separates into a creamy chylomicron layer floating on a still milky lower layer reflecting pre-beta lipoproteins.

Like all the other hyperlipoproteinaemias it may be secondary to a variety of disorders, including nephrosis, hypothyroidism, diabetes, keto-acidosis and excess alcohol consumption.

Highly levels of lipids may be associated with eruptive xanthomata, lipaemia retinalis, and hepato-splenomegaly. Attacks of abdominal pain analogous to those in Type I and IV hyperlipoproteinaemia may occur. The postheparin lipolytic activity is often within the normal range. Whether or not Type $\mathrm{V}$ hyperlipoproteinaemia produces arterial disease is not clear.

Treatment can be effective but may prove difficult. Reduction to ideal weight if indicated often produces a striking fall in the lipid levels with regression of the associated clinical features. Clofibrate may contribute to control the plasma lipid levels. Although the familial types of hyperlipoproteinemia are quite common in the unselected population secondary hyperlipoproteinemias associated with common diseases are much mor often. Out of these and for the rest of my time I should like to discuss some aspects of hyperlipoproteinemia in liver disease and one form of a secondary hypolipoproteinemia.

Hyperlipoproteinemia accompanying obstructive jaundice is well known for many years. Flint observed more than a century ago an increased blood cholesterol concentration in this group of patients. More recent studies have well documented that the characteristic elevation of unesterified cholesterol and phospholipids in patients with obstructuve jaundice are due to the presence of a low density lipoprotein of abnormal composition and properties. This abnormal plasma lipoprotein has been designated lipoprotein $\mathrm{x}$ (LP-X). The protein lipid composition of the isolated LP-X is unique and 
consists of $6 \%$ protein, $65 \%$ phospholipids, $23 \%$ unesterified cholesterol, $2 \%$ cholestrol esters and $3 \%$ triglycerides. Among bile acids present in LP-X (2-3\% by wt.) lithocholic acid, known to be liver toxic, is represented in relatively high amounts. The protein mojety of LP-X consists of a combination of $40 \%$ albumin and $60 \%$ apo $\mathrm{C}$. In the electronmicroscope LP-X appears as a spherical particle with a diameter ranging from $300-700 \mathrm{~A}$, and in contrast to normal lipoproteins with a strong tendency to aggregate and to form discs. It consists of a phospholipid, cholesterol, apo C wall. The albumin portion of LP-X seems to be masked in the core of the native particle. It has been proposed that the specific combination of apo $\mathrm{C}$ and albumin plays the important role in maintaining the structural integrity of this lipoprotein particle characterized by the high phospholipid-protein ratio of 11 .

A sensitive test system for determining selectively LP-X has recently been developed in our laboratory. When this technique was used, a significance of $p 0.001$ was found for the LP-X test to demonstrate or exclude cholestasis. In this respect the LP-X test is superior to all known blood chemical parameter.

Independent of the occurance of LP-X, various liver disorders are often associated with decreased concentrations of serum $\alpha$ and pre- $\beta$ lipoproteins, when lipoprotein electrophoresis is employed as criterion.

Further characterization of these two classes of plasma lipoproteins, however, clearly demonstrated that the decreased concentration of HDL is primarily due to an impaired lipid binding capacity of apo A resulting in an abnormally high protein lipid ratio and in severe cases a lack of neutral lipids. In contrast to normal $\alpha$ lipoproteins, this fraction does not stain for lipids, but shows two distinct and non identical precipitin bands on immunoelectrophoresis and immunodiffusion. The isolated VLDL from patients with liver disorders reveal a regular particle size and a protein lipid ratio close to normal, but develop $\beta$ mobility on electrophoresis. Analysis of the protein moieties of this fraction indicated a lack of apo A. It therefore is suggested that disturbed liver function leads to the synthesis of an altered apo A resulting in $\alpha$ lipoproteins with dissociated apo A peptides and in very low density lipoproteins devoid of apo A. Both findings may be explained by an impaired capacity of this apolipoprotein A to bind neutral lipids. These alterations may again normalize depending on the successful course of therapy. Disturbances of the thyroid gland are also known to influence the plasma lipid values. Hypothyroidism leads to an increase of $\beta$-lipoproteins and hypercholesterolemia. Hyperthyroidism is followed by hypocholesterolemia, but not by a decrease of the $\beta$ lipoproteins when lipoproteinelectrophoresis is applied as criterion. In patients with hyperthyroidism another abnormal lipoprotein could recently been isolated which migrates as $\beta$-lipoproteins but sediments at a density of $\mathrm{d} 1.063 \mathrm{~g} / \mathrm{ml}$. Normally the $\mathrm{d} 1.063$ bottom fraction only contains $\alpha$-lipoproteins. We have designated this abnormal lipoprotein " $\beta$-HDL". The protein lipid composition of $\beta$-HDL is distinguished by a high protein lipid ratio, a high content of protein and a low content of cholesterol in comparison to normal $\beta$-lipoproteins from the LDL fraction. The phospholipids and trigylcerides are present in the same concentrations as in normal $\beta$-lipoproteins. It is not possible to enrich the $\beta$-HDL with lipids. Under identical experimental conditions this is however possible with apo $\mathrm{A}$ and partially delipidized $\beta$ lipoproteins. From this it may be concluded that it is the protein compound of $\beta$-HDL rather than the availability of lipids which determines the protein lipid composition of this abnormal plasma lipoprotein. We have recently shown that the protein portion of $\beta$-HDL consists of normal apo $\beta$ and a second protein compound different to all other apolipoproteins. The concentration of $\beta$-HDL in patients with hyperthyroidism goes parallel with the severity of the disease and is no longer demonstrable, following the successful treatment nor is it detectable in healthy subjects. The determination of $\beta$-HDL therefore may prove useful for the diagnosis of hyperthyroidism.

Although there is still much uncertainly about structural relations of the lipids to the 
proteins in the plasma lipoproteins, I feel and I am concluding from our progress in recent years, some of which I tried to discuss with you today that further emphasis on the characterization of the protein moieties of plasmalipoproteins, and on the lipolytic enzymes and on

\section{Questions to Dr. Seidel}

1) a) When hyperthyroid patients are treated by any drugs, is the amount of $\beta$-HDL decreased depending upon the state of the patients?

b) How do you usually measure $\beta$-HDL content?

2) Are there any other drugs except estrogen which can influence the synthesis of apolipoprotein?

(Haruo Nakamura, Keio Univ. Tokyo)

\section{Answer:}

1) After a successful treatment the abnormal $\beta$-HDL is no longer demonstrable in the patient plasma. The concentration follows the severity of the desease better than the $T_{3} / T_{4}$ index. In normals the abnormal $\beta$-HDL can not be detected.

2) The best way to quantitate the concentration $a / \beta-\mathrm{HDL}$ is to ultracentrifuge the patients plasma of the density of $1.07 \mathrm{~g} / \mathrm{ml}$. On the bottom fraction a cholesterol determination should be performed which will give the cholesterol value for the $a$-lipoproteins and the abnormal $\beta$-HDL.

Then polyanion precipitation is performed on this bottom fraction (Heparin: $\quad \mathrm{Cl}_{2}$ ) and on the filtrate containing the $a$-lipoproteins a second cholesterol determination should be performed. The difference between the first cholesterol determination ( $a$-lipoproteins $+\beta$ HDL) and the second cholesterol determination (only $a$-lipoproteins) will selected the cholesterol transported by the $\beta$-HDL. This value may be transerful to total $\beta$-HDL concentration using the factor 5 , resulting from the $20 \%$ content of cholesterol of the $\beta$-HDL. However, you may as well perform a normal lipoprotein dectrophoresin on the $1.07 \mathrm{~g} / \mathrm{ml}$ bottom fraction and then obtain a semi quantitave determination of the $\beta$-HDL by densitometric means. the interactions of both may provide a better understanding of lipid metabolism and the factors regulating plasma lipid concentrations. It is reasonable to anticipate that this may also help to get more inside in the mechanism of atherosclerosis.

3) As I know so fare this effect has only been demonstrated for androgens in dogs. But I fell it should be very much indicated to look into this aspect of lipid metabolism in move deteil.

\section{Question to Dr. Seidel}

We understand that low and very low density lipoproteins are washing as a cancer of lipids. However, I wonder high density lipoprotein is also washing only lipids career or not. How is your opinion?

(Tadao Yasugi, Nihon Univ. Tokyo)

\section{Answer:}

There is certainly an exchange between apolipoprotein from one density class to another density class. Since there is not much of a phospholipid transport in the plasma from an organ to another organ (phospholipids over the predonin rat lipid compound of HDL) and since the action of the various proteins from the HDL fraction on the lipolytic enzymes has been demonstrated. I feel that the major fraction of the HDL-lipoproteins is involved in the metabolism of plasma lipoproteins rather than in the transport of lipids.

\section{Questions to Dr. Seidel}

1) I have an interest in the fact that the structures of $\beta$-lipoprotein in Type II hyperlipoproteinemia. Is there any experimental evidence that this fact promote the genesis of Atherosclerosis?

2) I want to know the Immunoassay method of lipoprotein for Type III.

(Hajime Orino, Tokyo Metropolitan

Geriatric Hspital)

Answer: 1)

1) This certainly most interesting question has not yet been studied. I feel the grant of Prof. Shimamoto should be the most qualifized team to look into this.

2) This has been published by us in clinica, 
Determination and Clinical Significance of Various Plasma Lipoprotein Pattern

chemica Acta \& Hurik in 1971. It utilizes the fact that the abnormal lipoprotein (floating $\beta$ ) in the type III patient contains in contrast to normal $\beta$-lipoproteins not only apolipoprotein $\beta$ but also apolipoprotein C. A simple immunobetrophaseic system using antibodies to apolipoprotein $\beta$ as reference and antisera to apoprotein $\mathrm{C}$ will provide the diagnosis of this type of hyperlipoproteinemia without the use of the ultracentifuge, which was a limiting factor for the exact typing of hyperlipoproteinemia. 